\title{
TORQue AERodinâmico, TORQue de Gradiente de GraVIDADE E TORQUE de RADIAÇÃo Solar ATUANTES EM SATÉLITES ESTABILIZADOS POR ROTAÇÃO
}

\author{
GABriel B. MotTA, MARia CECília ZANARDi.
}

\author{
Departamento de Matemática, FEG- UNESP \\ E-mails: gabriel_borderes@yahoo.com.br,cecilialfeg.unesp.br
}

\begin{abstract}
The aim of this paper is to analyze the effect of the aerodynamic torque, the solar radiation torque and the gravity gradient torque in the spin stabilized artificial satellite attitude. In order to do this a mathematical model to these torques is introduced and their components are included on the satellite spin motion equations. An analytical solution for these equations is proposed considering the sum of the mean components of each torque in an orbital period with the no zero z-component. This way the analytical solution is real for an orbital period, while the attitude variables are exchanged for new data computed through the analytical solution every orbital period. The proposed model has been applied for spin stabilized Brazilian satellite SCD1 and SCD2. The obtained data are compare with real data of the satellites provided by INPE (National Institute for Space Research).
\end{abstract}

Keywords— Rotation Dynamics, Aerodynamic Torque, Gravity Gradient Torque, Solar Radiation Torque

Resumo_- : O objetivo deste trabalho é analisar a influência do Torque Aerodinâmico, Torque de Radiação Solar e do Torque de Gradiente de Gravidade na atitude de satélites artificiais estabilizados por rotação. Para tanto, um modelo matemático para estes torque é apresentado e suas componentes incluídas nas equações do movimento rotacional do satélite. Uma solução analítica para as estas equações é proposta, considerando a soma dos componentes médios de cada torque em um período orbital, com a componente no eixo z não sendo nula. Assim esta solução analítica é válida para um período orbital, sendo que a cada período orbital as varáveis de atitude são atualizadas com o valor obtido da solução analítica proposta. O modelo proposto é aplicado para os dois Satélites Brasileiros de Coleta de Dados SCD1 e SCD2. Os dados obtidos são comparados com os dados reais dos satélites fornecidos pelo INPE (Instituto Nacional de pesquisas Espaciais).

Palavras-chave— Astrodinâmica e Mecânica Orbital, Torque Aerodinâmico, Torque de Gradiente de Gravidade, Torque de Radiação Solar

\section{Introdução}

A precisão de missões espaciais envolvendo satélites artificiais depende da posição e orientação em que estes se encontram no espaço, em relação a um sistema de referência fixo na Terra. Entretanto, a ação continua de torques causados por forças oriundas do meio onde os satélites orbitam, influencia significativamente o movimento destes, ou seja, a atitude e órbita dos satélites. Particularmente, a descrição do movimento rotacional de veículos espaciais é realizada através das equações de dinâmicas de Euler, as quais dependem das componentes desses torques. Portanto, é imprescindível, na análise de uma missão espacial, a avaliação das magnitudes destes torques ambientais (externos) em função da posição em que o veículo encontra-se em sua órbita.

Os torques ambientais que mais afetam a atitude são o Torque de Gradiente de Gravidade (TGG), o Torque de Pressão de Radiação Solar Direta (TRS), o Torque Aerodinâmico (TA) e os Torques Magnéticos. Neste trabalho os torques magnéticos não são incluídos sendo considerado apenas a influência dos demais torques no movimento rotacional de satélite estabilizados por rotação.

\section{Modelos Matemáticos para os Torques}

As equações do movimento rotacional de um satélite estabilizado por rotação dependem das componentes dos torques externos expressas em um sistema fixo no satélite, no qual o eixo $\mathrm{z}$ coincide com o eixo de rotação do satélite. Os modelos matemáticos adotados para o TGG, o TA e TRS aqui considerados estão apresentados em Chiaradia (2007), Chiaradia (2010) e Motta (2011), respectivamente. Bem como a determinação de seus componentes médios no sistema do satélite.

As forças gravitacionais surgem devido ao campo gravitacional gerado pelos planetas e corpos que possuem massa. O TGG ocorre devido ao gradiente de força gravitacional existente entre diferentes pontos do satélite, dependendo da distribuição de massa e da forma do satélite (Pisacane \& Moore, 1994), através dos momentos principais de inércia do satélite. Os três componentes médios deste torque no sistema fixo no satélite são discutidas detalhadamente em Chiaradia (2007), Pereira (2011) e Zanardi \& Pereira (2012).

As forças aerodinâmicas são criadas pela colisão das moléculas de ar rarefeito da alta atmosfera com a superfície do satélite. Quando o ponto de aplicação da resultante dessas forças não coincide com o centro de massa do satélite aparece o TA. Este torque é predominante em satélites de baixa altitude (com 
perigeu menor que quatrocentos quilômetros), uma vez que depende da quantidade de moléculas de ar na atmosfera da Terra (Zanardi, 2005). A magnitude deste torque está diretamente relacionada com a altitude em que o satélite se encontra em relação à superfície terrestre e do modelo adotado para a densidade atmosférica. Em termos da órbita do satélite, a atuação da força aerodinâmica diminui a velocidade do satélite no perigeu, causando, com o tempo, a diminuição do semi-eixo orbital, acarretando a circularização da órbita, diminuindo a vida útil do satélite (Pisacane \& Moore, 1994). Para o modelo do TA foram consideradas simplificações de modo a ser possível uma abordagem semi-analítica na busca de uma solução para as equações do movimento rotacional. Tais simplificações consideraram o TA associado apenas à força de arrasto, com o coeficiente de arrasto e a área de superfície transversal constantes, a atmosfera da Terra estática, a densidade atmosférica assumindo um valor numérico fornecido pelo modelo TD88 (Sehnal \& Pospísilová, 1988) e a margem estática fixa. Devido as simplificações realizadas o componente do TA no eixo $\mathrm{z}$ é nula, de modo que este torque não afeta a velocidade de rotação do satélite estabilizado por rotação. Detalhes deste modelo e a determinação de seus componentes médios são discutidos em Chiaradia (2010), Pereira (2011) e Zanardi \& Pereira (2012).

Em suas órbitas os satélites estão sujeitos à radiação proveniente de diversas fontes, sendo a mais relevante o sol. Pelo choque desta radiação com a superfície do satélite há uma troca de momento que dá origem a força de radiação solar, que por sua vez gera o TRS. A força de radiação solar pode ser obtida utilizando-se as taxas de reflexão difusa e especular dos fótons, da taxa de absorção dos fótons e do fluxo de radiação. Em Zanardi \& Vilhena de Morais (1999) é apresentado o modelo do TRS aplicado a um satélite cilíndrico que está sempre iluminado pelo sol, mais tarde em Motta (2011), o mesmo modelo é aplicado ao satélite estabilizado por rotação. Detalhes deste modelo e a determinação de seus componentes médios são discutidos em Motta (2011) e Zanardi \& Vilhena de Morais (1999). Devido as considerações adotadas, o componente do torque do eixo z é nulo.

\section{Equações do Movimento}

As equações do movimento de um satélite estabilizado por rotação são descritas pelas equações de Euler em termos da velocidade de rotação $(W)$, da ascensão reta $(\alpha)$ e declinação $(\delta)$ do eixo de rotação (Figura 1). Tais equações dependem dos componentes dos torques externos no sistema fixo no satélite e são dadas por (Kuga et. all, 1987 ):

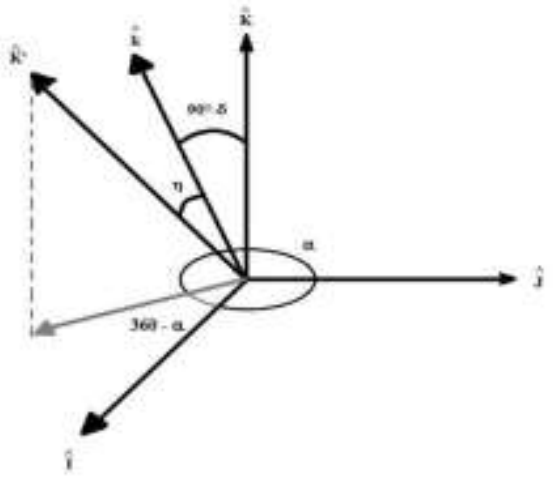

Figura 1: Sistema equatorial $(\hat{I} \quad \widehat{K})$, sistema fixo no satélite $\left(\begin{array}{ll}l & \hat{k}\end{array}\right)$, ascensão reta $(\alpha)$ e declinação $(\delta)$ do eixo de rotação $(W)$ (Motta, 2011)

Os principais efeitos dos torques no movimento rotacional podem ser determinados pelos seus componentes médios em um período orbital. Portanto no processo de integração analítico, considera se os valores numéricos para os componentes dos torques médios obtidos com os dados iniciais de atitude e órbita do satélite.

\section{Solução das Equações do Movimento}

No processo de integração das equações (1), (2) e (3) são utilizados os componentes médios da soma dos torques externos considerados, sendo que os componentes médios de cada torque são obtidos em Zanardi e Pereira (2011) e Motta (2011).

\subsection{Solução analítica para a Velocidade de rotação}

Da equação diferencial apresentada em (3), é possível se obter uma solução analítica para a velocidade de rotação válida para um período orbital.

Neste processo no $N_{z}$ é substituído por $N_{z m}$ que é a soma dos componentes médios no eixo $z$ do TA, TGG e TRS. Tanto $\mathrm{N}_{\mathrm{zm}}$ como $I_{z}$ são constantes em relação ao tempo, tem-se então: 
sendo o valor da velocidade de rotação no instante inicia.

Portanto os torques considerados causam uma variação linear na velocidade de rotação devido o componente dos torques externos no eixo $z$. Salienta-se que pelas considerações realizadas para o TA e o TRS, seus componentes são nulos no eixo $z$. Deste modo a variação linear em $W$ devido apenas ao TGG.

\subsection{Solução analítica para a declinação do eixo de rotação}

Utilizando (4) na equação diferencial apresentada em (2), é possível obter uma solução analítica para a declinação do eixo de rotação válida para um período orbital.

Aqui também $N_{y}$ é substituído por $N_{y m}$ que é a soma dos componentes médios no eixo $y$ do TA, TGG e TRS, sendo $N_{y m}, N_{z m}$ e $I_{z}$ admitidos constantes em relação ao tempo. Através de uma substituição simples em que se utiliza a solução para $W$, dada em (4), e em decorrência — $d W$, a equação (2) pode ser apresenta da forma:

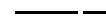

cuja a solução é dada por:

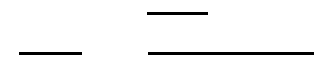

sendo o valor da declinação do eixo de rotação no instante inicial. Do mesmo modo que para a velocidade de rotação $W$, apenas o TGG contribui para a variação temporal de $\delta$. Saliente-se que a solução (6) só é válida quando $\mathrm{N}_{\mathrm{zm}}$ não é nulo.

\subsection{Solução analitica para a ascensão reta do eixo de rotação}

Aqui também $N_{x}$ é substituído por $N_{x m}$ que é a soma dos componentes médios no eixo $x$ do TA, TGG e TRS.

Substituindo agora a solução analítica da declinação do eixo de rotação, dada em (6), na equação associada com a ascensão reta do eixo de rotação, dada por (1) obtém-se: com $N_{x m}, N_{y m}, N_{z m} e I_{z}$ constantes. Onde $W$ é a função que depende explicitamente do tempo, da maneira apresentada em (4).

Para $\mathrm{N}_{\mathrm{zm}}$ não nulo, na integração da equação (6), inicialmente foi realizada uma substituição simples semelhante à utilizada na integração da equação (2), através da solução (4) e da relação (5):

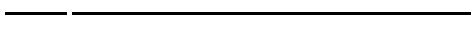

Realizando uma nova substituição onde se usa a a relação (5) e a solução (6), a equação fica:

Por meio do conceito de secante, realiza-se então os procedimentos necessários para a integração. Após a integração e as devidas substituições feitas, o comportamento temporal da ascensão reta é dado por:

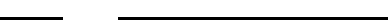

em que $\delta$ é apresentado em (6) e $\alpha_{0}$ é o valor da ascensão reta do eixo de rotação no instante inicial.

Assim a solução aqui obtida é valida quando o componente do torque externo no eixo $z$ não é nulo. Caso contrario, a solução analítica a ser determinada é similar a obtida em Motta(2011), quando apenas o TRS foi considerado, fazendo com que a velocidade de rotação permanecesse constante.

\section{Aplicações}

Para validar as soluções analíticas obtidas no item anterior são aqui utilizados os dados dos Satélites Brasileiros de Coleta de Dados SCD1 e SCD2, comparando os valores obtidos pela teoria para $W, \alpha$ e $\delta$. São apresentados os resultados para o erro de apontamento que é o deslocamento angular entre o eixo de rotação real e o eixo de rotação simulado, que pode ser determinado através dos ângulos $\alpha$ e $\delta$. Também é calculado o ângulo de aspecto solar, que é o ângulo entre o eixo de rotação e a direção satélite (Chiaradia, 2010). Para validar a solução analítica é calculado o desvio quadrático médio da diferença obtida entre os dados reais e os simulados, juntamente com o erro de apontamento e ângulo de aspecto solar. Todas as simulações foram realizadas com o software MATLAB e os dados dos satélites foram atualizados a cada 24 horas pelos dados fornecidos pelo CCS/INPE.

Os dados disponibilizados pelo INPE abrangeram um período de 40 dias, a partir do dia 24/07/93 para o SCD1 e a partir de 01/02/02 para o SCD2. Para a aplicação aqui realizada foram analisados 
previamente os resultados obtidos utilizando cada torque em Chiaradia (2007), Chiaradia (2010) e Motta (2011).

Nas figuras de 2 a 11 são apresentadas a diferença na ascensão reta, declinação do eixo de rotação, velocidade de rotação, ângulo de aspecto solar e erro de apontamento entre dados reais e calculados, para SCD1 e SCD2 respectivamente. Observa-se um bom comportamento em todos os parâmetros avaliados com apenas alguns pontos excedendo a exigência do INPE que é de $0,5^{\circ}$ ou $0,5 \mathrm{rpm}$, exceto pela diferença na ascensão reta do eixo de rotação no SCD1 que ultrapassa a exigência em muito e durante muito tempo.

$\mathrm{Na}$ Tabela 1 verifica-se o mesmo comportamento em observado nas figuras através do erro quadrático médio, nota-se que somente a ascensão reta do eixo de rotação no SCD1 ultrapassa a exigência.

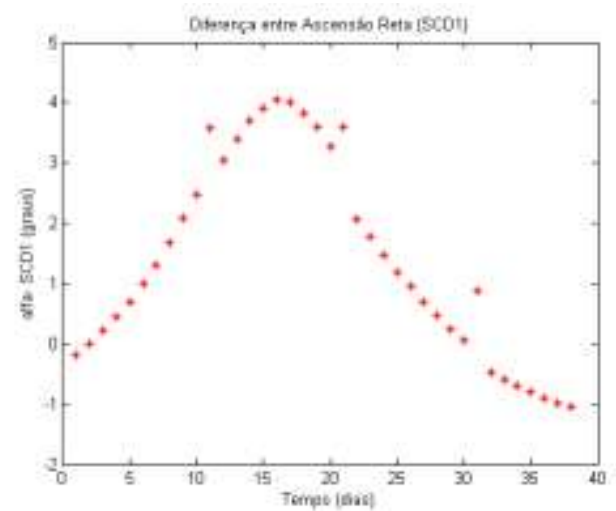

Figura 2: Diferença entre a ascensão reta do eixo de rotação obtida através de implementação e fornecida pelo CCS/IMPE do SCD1.

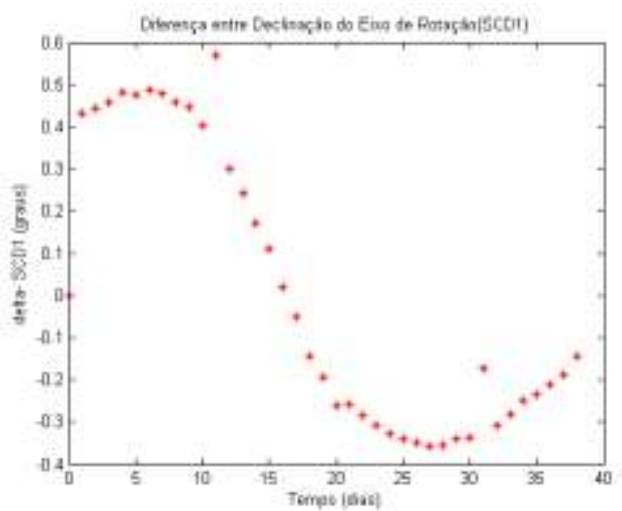

Figura 3: Diferença entre a declinação do eixo de rotação obtida através de implementação e fornecida pelo CCS/IMPE do SCD1.

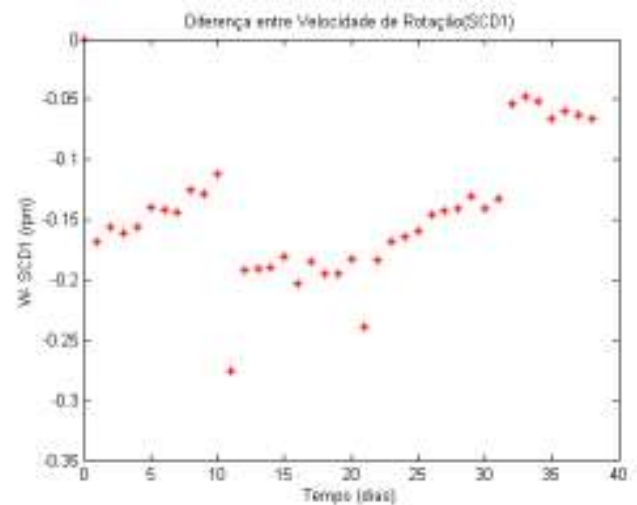

Figura 4: Diferença entre a velocidade de rotação obtida através de implementação e fornecida pelo CCS/IMPE do SCD1.

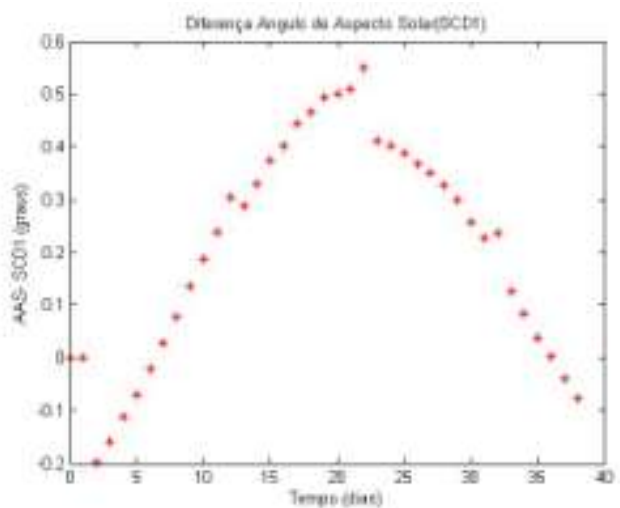

Figura 5: Diferença entre o ângulo de aspecto solar obtido através de implementação e fornecido pelo CCS/IMPE do SCD1.

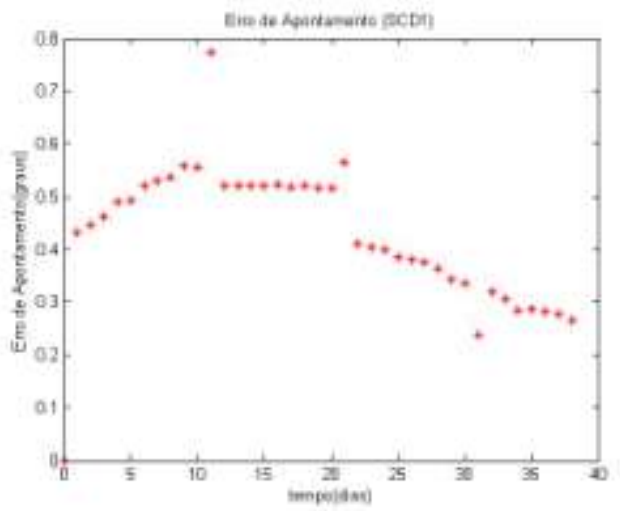

Figura 6: Erro de apontamento obtido através de implementação e fornecido pelo CCS/IMPE do SCD1.

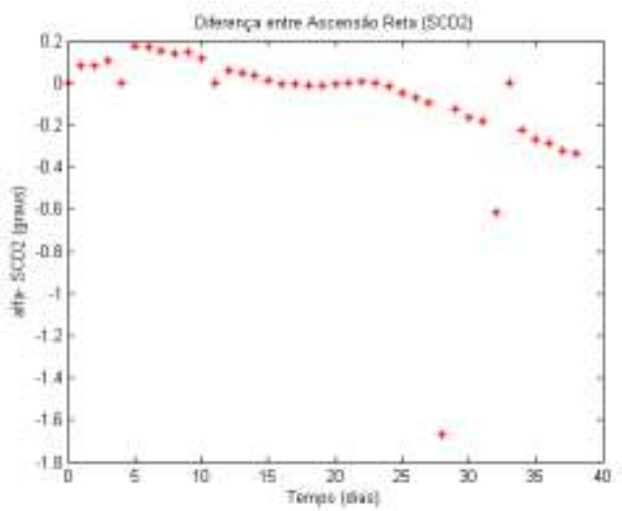

Figura 7: Diferença entre a ascensão reta do eixo de rotação obtida através de implementação e fornecida pelo CCS/IMPE do SCD2. 


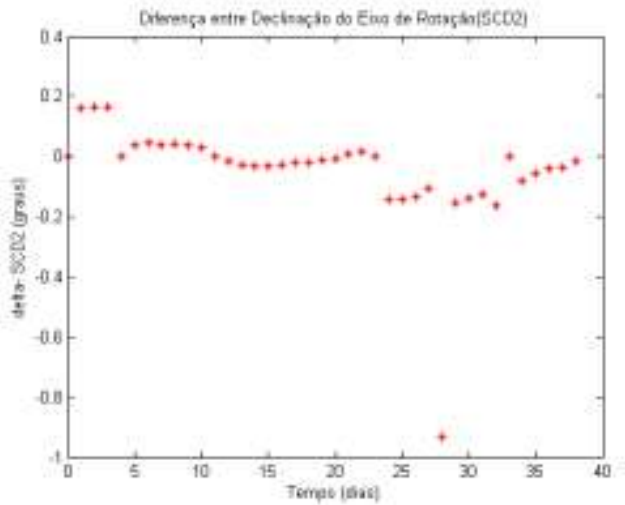

Figura 8: Diferença entre a declinação do eixo de rotação obtida através de implementação e fornecida pelo CCS/IMPE do SCD2.

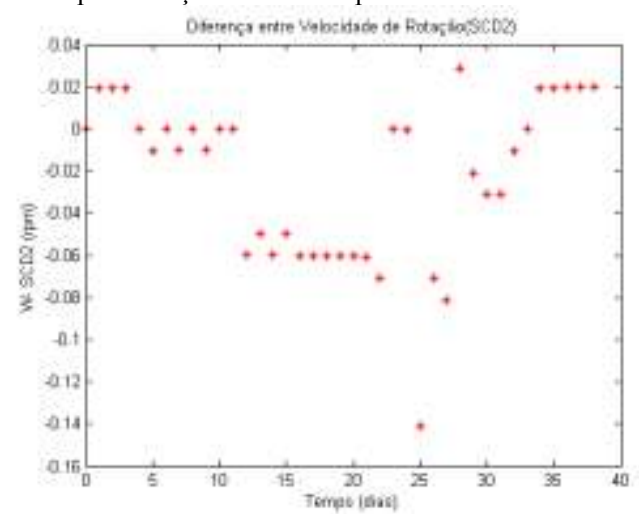

Figura 9: Diferença entre a velocidade de rotação obtida através de implementação e fornecida pelo CCS/IMPE do SCD2.

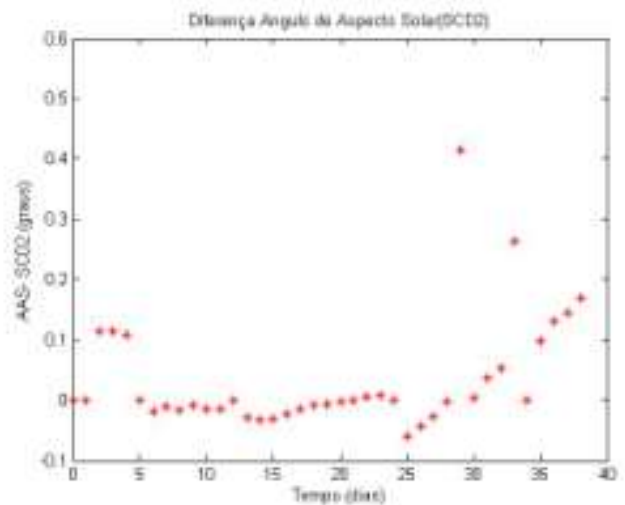

Figura 10: Diferença entre o ângulo de aspecto solar obtido através de implementação e fornecido pelo CCS/IMPE do SCD2.

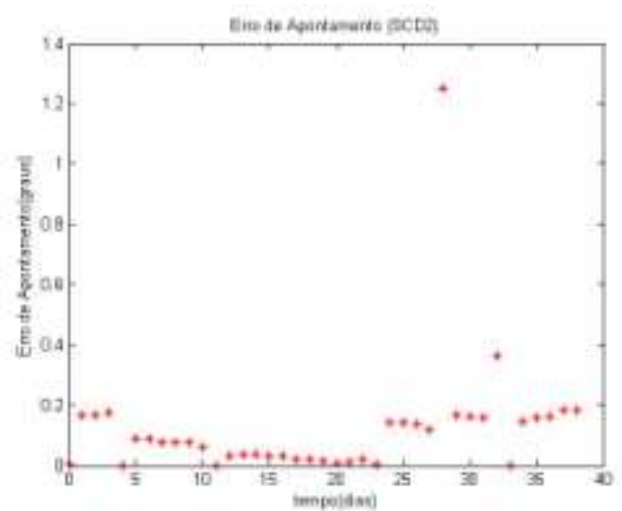

Figura 11: Erro de apontamento obtido através de implementação e fornecido pelo CCS/IMPE do SCD2.
Tabela 1: Desvio Quadrático Médio para o SCD1 e SCD2.

\begin{tabular}{|l|c|c|}
\cline { 2 - 3 } \multicolumn{1}{c|}{} & SCD1 & SCD2 \\
\hline $\begin{array}{l}\text { Ascenção Reta do Eixo } \\
\text { Declinação } \\
\text { Eixo do Rotação }\end{array}$ & 1,6740 & 0,3067 \\
$\begin{array}{l}\text { Velocidade de } \\
\text { Rotação }\end{array}$ & 0,3335 & 0,1666 \\
$\begin{array}{l}\text { Ângulo de } \\
\begin{array}{l}\text { Aspecto Solar } \\
\text { Erro de Apontamento }\end{array}\end{array}$ & 0,0573 & 0,0387 \\
\hline
\end{tabular}

\section{Conclusão}

Os resultados obtidos com os dados dos satélites SCD1 e SCD2 apontam para uma concordância entre a solução teórica e o comportamento real do movimento rotacional dos satélites. Foram obtidos bons resultados sendo que a precisão se encontra, em maior parte, dentro da exigência de $0,5^{\circ}$ ou $0,5 \mathrm{rpm}$ exigidas pelo INPE.

A solução analítica é válida para um período orbital e a implementação numérica foi realizada com a atualização diária dos dados de atitude e órbita fornecidos pelo INPE. No entanto é também importante validar esta teoria com a não atualização diária dos dados do satélite.

Saliente-se também que os torques magnéticos (Torque Magnético Residual e Torque devido as correntes de Focault) não foram incluídos nas equações do movimento mas contribuem de forma significativa na atitude do satélite (Kuga et al., 1987). A relevância destes torques esta na dependência de um deles da velocidade de rotação, que causa um decaimento exponencial na velocidade de rotação, e em na magnitude de ambos. Deste modo é importante a inclusão e análise da influência destes torques.

\section{Agradecimentos}

Os autores agradecem a FAPESP (proc. 012/21023-6) e a CAPES pelo apoio financeiro.

\section{Referências Bibliográficas}

Chiaradia, C. E. (2007). "Influência do Torque de Gradiente de Gravidade na Atitude de Sat. Art. estabilizados por rotação", Trabalho de Graduação.UNESP - Campus de Guaratinguetá.

Chiaradia, J. E. (2010). "Torque Aerodinâmico e o Movimento Rotacional de Satélites Artificiais Estabilizados por Rotação", Trabalho de Graduação.UNESP - Campus de Guaratinguetá.

Kuga, H. K.; Silva, W. C. C.; Guedes, U. T. V. (1987). "Dinâmica de atitude para satélites estabilizados por rotação". São José dos Campos - SP, Relatório Técnico do INPE, INPE-4403NTE/275. INPE.

Motta, G. B. (2011). "Satélites Estabilizados por Rotação e Torque de Radiação Solar Direta", 
Trabalho de Graduação.UNESP - Campus de Guaratinguetá.

Pereira, A. J. (2011) "Satélites Estabilizados por Rotação: Torques Externos e Ângulo de Aspecto Solar". Dissertação de Mestrado, UNESP - Campus de Guaratinguetá.

Pisacane, V. L., Moore, R. C. (1994). "Fundamentals of Space System", New York: Oxfort University Press.

Sehnal, L., Pospísilová, L. (1988). "Thermospheric model TD88", Preprint No. 67 of the Astronomical Institute of Czechoslovakian Academy of Sciences.

Zanardi, M. C., Vilhena de Moraes, R. (1999) "Analytical and Semi-analytical Analysis of an Artificial Satellite's Totaional Motion", Cels. Mech. And Dyn. Astron., v. 75, no. 4, pp. 227-250. DOI: $10.1023 / \mathrm{A}: 1008358801859$

Zanardi, M. C.(2005). "Dinâmica da Atitude de Satélites Artificiais", Tese de Livre Docência, UNESP - Campus de Guaratinguetá.

Zanardi, M. C., Pereira, A. J., (2012) "Spin stabilized satellite's attitude analytical propagation", Journal of Aerospace Engineering, Sciences and Applications, v. IV, p. 31 - 41 . 